\title{
ORIGINAL ARTICLE An evolutionary history of the selectin gene cluster in humans
}

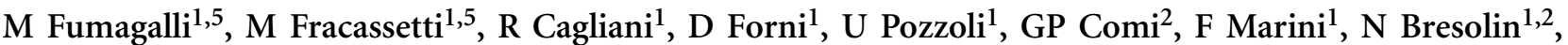 \\ M Clerici ${ }^{3,4}$ and M Sironi ${ }^{1}$
}

Molecules involved in leukocyte trafficking have a central role in the development of inflammatory and immune responses. We performed $F_{\mathrm{ST}}$ analysis of the selectin cluster, as well as of SELPLG, ICAM1 and VCAM1. Peaks of significantly high population genetic differentiation were restricted to two regions in SELP and one in SELPLG. Resequencing data indicated that the region covering SELP exons 11-13 displays high nucleotide diversity in Africans and Europeans (CEU), and a high level of withinspecies diversity compared with inter-specific divergence. Analysis of inferred haplotypes revealed a complex phylogeny with two deeply separated clades that coalesce at $\sim 3.5$ million years (MY) plus a minor clade with a TMRCA (time to the most recent common ancestor) of $\sim 2.2 \mathrm{MY}$. A splicing assay indicated no haplotype-specific effect on SELP exon 14 inclusion. These data are consistent with a model of multiallelic balancing selection; single-nucleotide polymorphism analysis indicated that the Val640Leu variant represents a likely selection target. In populations of Asian ancestry a distinct haplotype, possibly carrying regulatory variants, has been driven to high frequency by positive selection. No deviation from neutrality was observed for the $S E L P L G$ region. Resequencing of SELP in chimpanzees revealed a haplotype phylogeny with extremely deep basal branches, suggesting either long-standing balancing selection or ancestral population structure. Thus, SELP has experienced a complex selective history, possibly as a result of local adaptation. Variants in the gene have been associated with autoimmune and cardiovascular diseases. Association studies would benefit from both taking the complex SELP haplotype structure into account and from analysis of possible regulatory variants in the gene.

Heredity (2012) 109, 117-126; doi:10.1038/hdy.2012.20; published online 2 May 2012

Keywords: selectin gene cluster; SELP; balancing selection; local adaptation

\section{INTRODUCTION}

Leukocyte interaction with the vascular endothelium has a central role in the development of inflammatory and immune responses. Thus, adhesion molecules involved in leukocyte trafficking have received considerable interest, especially in relation to the development of human diseases. The most well-studied molecules are the selectins, ICAM1 (intercellular adhesion molecule 1) and VCAM1 (vascular cell adhesion molecule 1).

Selectins are a family of C-type lectins, and humans express three family members, namely P-, E- and L-selectin, encoded by genes (SELP, SELE and SELL) located in cluster on chromosome 1. By binding to their ligands, selectins have a major role in leukocyte tethering and rolling on activated epithelia, thus favouring their extravasation and migration to inflamed tissues. The prefix for the three selectins carries a hint at their main expression site: L- and E-selectins are expressed on most leukocytes and on activated endothelial cells, respectively, whereas P-selectin is stored in platelet alpha granules and endothelial Weibel-Palade bodies (Ley, 2003). The three selectins have a common ligand, encoded by SELPLG. Exposure to different pro-inflammatory and pro-thrombotic stimuli determines the rapid translocation of SELP to the cell surface where, through interaction with SELPLG, it promotes adhesion and rolling of both leukocytes and platelets, as well as their heterotypic aggregation (Ley, 2003). Inflammatory cytokines also induce E-selectin transcription, whereas L-selectin is constitutively expressed (Ley, 2003). Experiments in knockout mouse models, as well as data from rare human conditions where leukocyte binding to selectins is impaired, indicate that SELP, SELL and SELE have a central role in the trafficking of innate immunity cells, T lymphocytes, and platelets (Ley, 2003).

ICAM1 and VCAM1 belong to the immunoglobulin superfamily; inflammatory cytokines also trigger the expression of both ICAM1 and VCAM1 on endothelial cells to increase leukocyte adherence and migration (Golias et al., 2007).

Selectins, as well as ICAM1 and VCAM1, also exist in soluble forms. The circulating amount of P-selectin and ICAM1 is influenced by polymorphisms located at their respective loci, and by variants in ABO (Miller et al., 2004; Reiner et al., 2008; Barbalic et al., 2010).

Leukocyte extravasation and tissue infiltration are necessary for efficient immune response against invading pathogens; yet, these processes may also result in tissue damage and promote chronic

${ }^{1}$ Scientific Institute IRCCS E. Medea, Bioinformatics Lab., Bosisio Parini (LC), Italy; ${ }^{2}$ Department of Neurological Sciences, Dino Ferrari Centre, University of Milan, Fondazione Ca' Granda IRCCS Ospedale Maggiore Policlinico, Milano, Italy; ${ }^{3}$ Department of Biomedical Sciences and Technologies LITA Segrate, University of Milan, Milano, Italy and ${ }^{4}$ Department of Molecular Medicine, Fondazione Don C. Gnocchi, IRCCS, Milano, Italy

${ }^{5}$ These authors equally contributed to this work.

Correspondence: Dr M Sironi, Scientific Institute IRCCS E. Medea, Bioinformatic Lab, Via don L. Monza 20, Bosisio Parini (LC) 23842, Italy.

E-mail: manuela.sironi@bp.Inf.it

Received 31 October 2011; revised 7 February 2012; accepted 19 March 2012; published online 2 May 2012 
inflammatory states. These observations suggest on one hand that genes encoding adhesion molecules may represent natural selection targets, as is the case of several immune response loci (Sironi and Clerici, 2010); on the other hand, genetic variation at these loci might protect from or predispose to autoimmune or chronic inflammatory conditions. Indeed, polymorphisms in SELP and SELE have been associated with susceptibility to systemic lupus erythematosus (Jacob et al., 2007), whereas variants in SELL and SELE predispose to IgA nephropathy (Watanabe et al., 2006). SELP also represents a risk locus for atopy (Bourgain et al., 2003).

The increasing availability of large-scale genetic diversity data, which are being produced by next-generation sequencing platforms, opens unprecedented possibilities to the study of natural selection signatures along extended genomic regions. Still, the nature of these data also requires the development of new strategies to infer selective patterns. Here, we aimed to provide a description of the evolutionary history of the selectin gene cluster, by integrating low-coverage genome-wide resequencing data with classic population genetics analyses. Similarly, the genes encoding ICAM1, VCAM1 and SELPLG were analyzed. Results indicate that a complex selective scenario in distinct human populations has shaped the genetic diversity of the SELP gene regions where autoimmune disease risk alleles are located.

\section{MATERIALS AND METHODS}

\section{Samples and sequencing}

Human genomic DNA from HapMap subjects (20 individuals of Asian descent) was obtained from the Coriell Institute for Medical Research. All analyzed regions were PCR-amplified and directly sequenced; primer sequences are available upon request. PCR products were treated with ExoSAP-IT (USB Corporation, Cleveland, OH, USA), directly sequenced on both strands with a Big Dye Terminator sequencing Kit (v3.1 Applied Biosystems, Foster City, CA, USA) and run on an Applied Biosystems ABI 3130 XL Genetic Analyzer (Applied Biosystems). Sequences were assembled using AutoAssembler version 1.4.0 (Applied Biosystems), and inspected manually by two distinct operators. The genomic DNA of nine Pan troglodytes was obtained from the Gene Bank of Primates, Primate Genetics, Göttingen, Germany (http://dpz.eu/index.php). These samples have been shown to belong to the Pan troglodytes verus subspecies (Cagliani et al., 2012). Primer pairs are available upon request.

\section{Population genetic analyses}

Tajima's $D$ (Tajima, 1989), Fu and Li's $D^{*}$ and $F^{*}$ (Fu and Li, 1993) statistics, as well as diversity parameters $\theta_{\mathrm{W}}$ (Watterson. 1975) and $\pi$ (Nei and Li. 1979) were calculated as previously described (Fumagalli et al., 2009). Calibrated coalescent simulations were performed using the cosi package (Schaffner et al., 2005) and its best-fit parameters for African American (AA), CEU and Asian (AS) populations with 10000 iterations. Coalescent simulations were conditioned on mutation rate and recombination rate was derived from University of California Santa Cruz (UCSC) tables (http://genome.ucsc.edu/, snpRecombRateHamap table).

The maximum-likelihood-ratio Hudson-Kreitman-Aguadé (MLHKA) test was performed using the MLHKA software (Wright and Charlesworth, 2004), as previously proposed (Fumagalli et al., 2009). For human populations, 16 reference loci were randomly selected among loci shorter than $20 \mathrm{~kb}$ that have been resequenced in $\mathrm{AA}, \mathrm{CEU}$ and $\mathrm{AS}$ by The National Institute of Environmental Health Sciences (NIEHS); the only criterion was that Tajima's $D$ did not suggest the action of natural selection (that is, Tajima's $D$ is higher than the 5th and lower than the 95th percentiles in the distribution of NIEHS genes). As for chimpanzee, the MLHKA was performed using the 16 resequenced regions as reference loci.

The DIND (derived intra-allelic nucleotide diversity) test was performed as previously proposed (Barreiro et al., 2009). Significance thresholds for each MAF interval were computed via coalescent simulations incorporating a demographic model (Schaffner et al., 2005).

Genotype data for $5 \mathrm{~kb}$ regions from 238 resequenced human genes were derived from the NIEHS SNPs Program web site (http://egp.gs. washington.edu). In particular, we selected genes that had been resequenced in populations of defined ethnicity including CEU, AA and AS (NIEHS panel 2). The iES (integrated extended haplotype homozygosity) score was calculated as previously described (Tang et al., 2007).

Details on $F_{\mathrm{ST}}$ statistic calculation among continental groups in the HGDPCEPH panel are available as Supplementary Methods. Genotype information was obtained for the SELP regions and for 2000 randomly selected RefSeq genes. Sliding-window analysis was performed on overlapping 20 singlenucleotide polymorphism (SNP) windows moving with a step of 5 SNPs.

\section{Haplotype analysis and TMRCA calculation}

Haplotypes were inferred using PHASE (version 2.1) (Stephens and Scheet, 2005). Details on haplotype analysis and TMRCA calculation are available as Supplementary Methods.

\section{Minigene construction, transfection and transcript analysis}

The genomic DNA of NA10851 HapMap subject (heterozygous for haplotypes belonging to the two main clades) was used as a template to PCR amplify a genomic fragment encompassing SELP exons 11-16. PCR amplifications were performed with high-fidelity polymerase ( $P f u$ DNA Polymerase, Promega Corporation, Madison, WI, USA). The $\sim 6500 \mathrm{bp}$ PCR products were cloned in the pDisplay expression vector (Invitrogen, Carlsbad, CA, USA) to generate the pDisplay-SELP-HapA and pDisplay-SELP-HapB constructs. The two plasmids were checked by direct DNA sequencing using primers located on the pDisplay vector (HA-For: 5'-GATGTTCCAGATTATGCTG-3'; MYC-Rev: $5^{\prime}$-CAGATCCTCTTCTGAGATG-3').

HeLa cells were maintained in DMEM medium supplemented with $10 \%$ fetal-calf serum and transfected using Lipofectamine-2000 (Invitrogen) in accordance with the manufacturer's instructions. Cells were harvested $48 \mathrm{~h}$ after transfection and total RNA was extracted by TRIzol reagent (Invitrogen), following the manufacturer's protocols. Purified RNA was reverse transcribed to cDNA using random hexamers and Ready-To-Go You-Prime First-Strand Beads (GE Healthcare, Little Chalfont, Buckinghamshire, UK). RT-PCR was performed with SELP primers located in exon 12 and 16. PCR products were separated on a $2 \%$ agarose gel.

\section{RESULTS}

Sliding-window analysis of the selectin cluster

In humans, three selectin genes (SELP, SELL and SELE) are located in $\mathrm{a} \sim 150 \mathrm{~kb}$ region on chromosome 1 . In order to analyze population genetic differentiation along the selectin cluster, we exploited data from the 1000 Genomes Pilot project deriving from the low-coverage whole-genome sequencing of 179 individuals with different ancestry (1000 Genomes Project Consortium et al., 2010). In particular, individuals from three distinct ethnic groups have been sequenced: CEU, Yoruba from Nigeria (YRI) and Japanese plus Chinese (East Asian, AS). Using these data we calculated $F_{\mathrm{ST}}$ (Wright, 1950) in sliding-windows moving along the chromosome 1 genomic region where SELE, SELL and SELP are located. As an empirical comparison, the same procedure was applied to 2000 randomly selected human genes, so as to calculate percentiles of genetic differentiation for pairwise population comparisons. As shown in Figure 1, prominent peaks in $F_{\mathrm{ST}}$ occur within the SELP gene region. Specifically, AS/YRI population genetic differentiation exceeds the 95th percentile in two regions covering exons $9-10$ and $11-13$. In these two regions $F_{\mathrm{ST}}$ is also high for CEU/AS and YRI/CEU comparisons (Figure 1). The 1000 Genomes Pilot project data have an advantage over other data sets (for example, HapMap and the HGDP-CEPH panel) because they do not rely on the genotyping of selected SNPs, resulting in a lessmarked bias towards intermediate frequency variants. Still, these data suffer from lack of power for rare variants (1000 Genomes Project Consortium et al., 2010), and possible biases within repetitive elements (Cagliani et al., 2012). Thus, we repeated $F_{\mathrm{ST}}$ analysis using 
both HapMap data and genotype information from the HGDP-CEPH panel: we obtained results very similar to those from the 1000 Genomes Pilot project data (Figure 1 and Supplementary Figure 1). These were also confirmed by $F_{\mathrm{ST}}$ analysis using Sanger resequencing data for SELP, SELL and SELE from the SeattleSNPs Variation Discovery Resource (Supplementary Figure 2). Finally, we calculated the iES (Tang et al., 2007) along the selectin cluster without identifying regions with unusual score (Supplementary Figure 3). These results warrant further analysis on the selection pattern of the two SELP regions, hereafter referred to as $S E L P_{\text {ex } 9-10}$ and $S E L P_{\text {ex11-13. }}$.

\section{Sliding-window analysis of SELPLG, ICAM1 and VCAM1}

The same approach described above was applied to analyze $F_{\mathrm{ST}}$ along SELPLG, ICAM1 and VCAM1. No unusual $F_{\mathrm{ST}}$ value was observed in ICAM1 and VCAM1 using data from the 1000 Genomes Pilot project; conversely a region of high genetic differentiation between YRI and AS was observed in the $5^{\prime}$ portion of SELPLG (hereafter referred to as $S E L P L G_{5^{\prime}}$ ) (Figure 2). High genetic differentiation was not detected using the HGDP-CEPH panel data (Figure 2), possibly because of the small number of SNPs genotyped in SELPLG; similarly, analysis of HapMap SNPs (Supplementary Figure 4) revealed no unusual $F_{\mathrm{ST}}$ value in the gene, nor did data derived from SeattleSNPs (Supplementary Figure 5). Yet, in this latter case the analysis was restricted to CEU and AA, as no AS individual was resequenced by the Program.

Population genetics of SELP and SELPLG in human populations In order to perform further population genetics analyses we turned to Sanger resequencing data. As mentioned above, the SELP and SELPLG transcription units have been fully resequenced in CEU and AA by the
Seattle SNPs Variation Discovery Resource. As for AS, we resequenced $S E L P_{\text {ex9-10 }}$, SELP $P_{\text {ex11-13 }}$ and SELPLG $5_{5^{\prime}}$ in 20 HapMap AS individuals. These data were used to calculate $\theta_{\mathrm{W}}$, an estimate of the expected per site heterozygosity (Watterson, 1975) and $\pi$, the average number of pairwise sequence nucleotide differences between haplotypes (Nei and $\mathrm{Li}, 1979$ ). In order to compare the values we obtained for the SELP and SELPLG regions, we calculated $\theta_{\mathrm{W}}$ and $\pi$ for $5 \mathrm{~kb}$ windows (hereafter referred to as reference windows) deriving from 238 genes resequenced by the NIEHS Program in the same populations; the percentile rank corresponding to the SELP and SELPLG regions in the distribution of reference windows is reported in Table 1 . No exceptional $\theta_{\mathrm{W}}$ or $\pi$ value was obtained for $S E L P_{e x 9-10}$ and $S E L P L G_{5^{\prime}}$ in the three populations. Conversely, both indexes were extremely high in SELP $P_{\text {ex11-13 }}$ in AA and $\mathrm{CEU}$, although in this latter population the rank of $\pi$ did not reach the 95th percentile. Peaks of nucleotide diversity in this region were also obtained for YRI using a sliding-window approach with the 1000 Genomes Pilot Project data (Supplementary Figure 6)

High nucleotide diversity might be suggestive of balancing selection, as neutral variants tend to be maintained with the selected allele(s). In order to formally rule out the possibility that the excess of nucleotide diversity is due to higher local mutations rates, we applied a MLHKA test (Wright and Charlesworth, 2004). This is based on the concept whereby, under neutral evolution, the amount of within- and between-species diversity is expected to be similar for all loci in the genome. Therefore, the test compares polymorphism and divergence levels for a region of interest with those calculated for other neutrally evolving genomic segments. Sixteen reference loci were used for the analysis (Fumagalli et al., 2009), and the results for $S E L P_{\text {ex11-13 }}$ indicated a significant excess

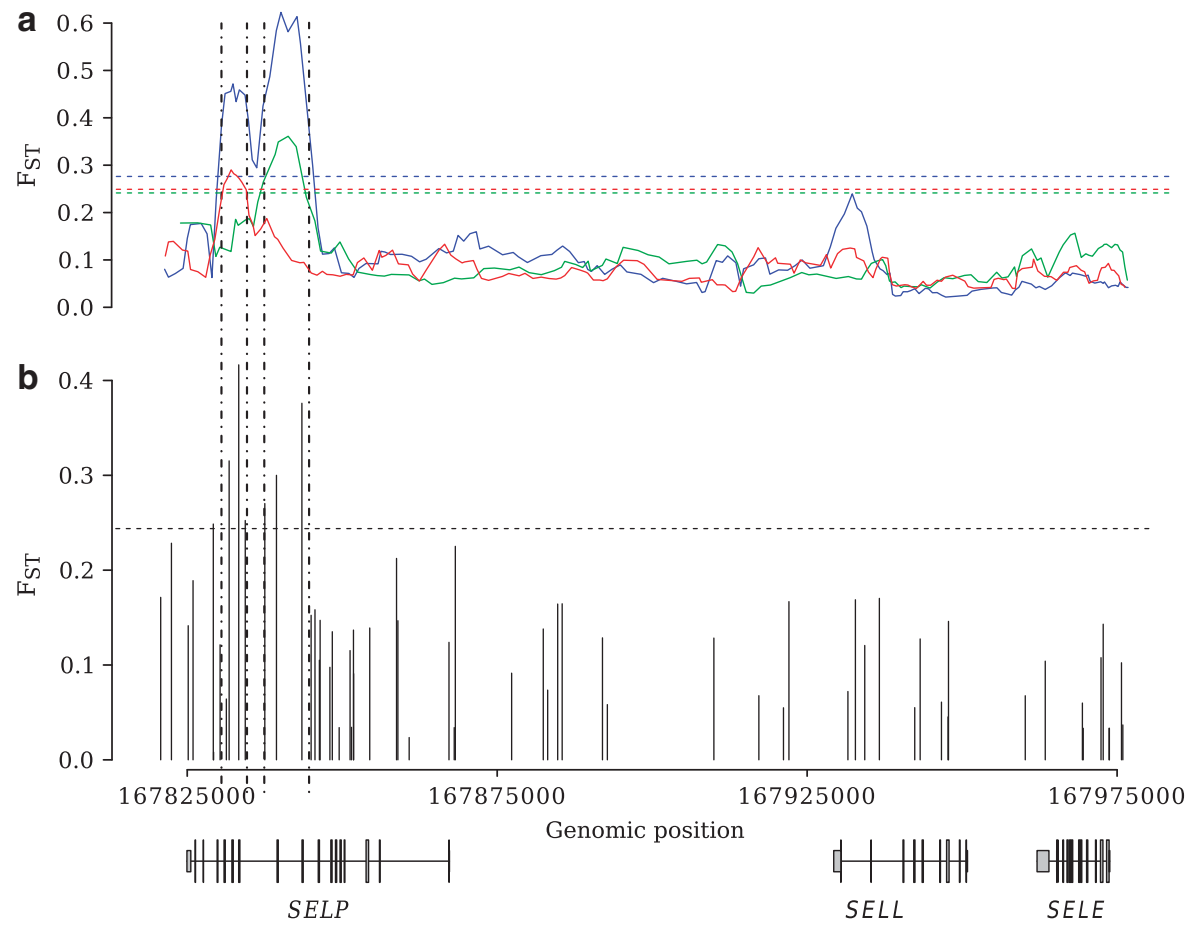

Figure 1 Sliding-window analysis of $F_{\mathrm{ST}}$ along the selectin cluster. (a) Data from the 1000 Genomes Pilot Project were used to calculate $F_{\mathrm{ST}}$ in sliding windows of 20 SNPs moving along the selectin gene cluster with a step of 5 SNPs. Color codes refer to population comparisons: red, YRI/CEU; blue, YRI/AS; green, CEU/AS. Horizontal dashed lines represent the 95th percentile in the distribution of $F_{\text {ST }}$ calculated for sliding windows deriving from 2000 randomly selected human genes. (b) The location of SNPs genotyped in the HGDP-CEPH panel is reported with the value of $F_{\mathrm{ST}}$ calculated across continental groups. The horizontal line corresponds to the 95th percentile in the distribution of all SNPs genotyped in the panel. 

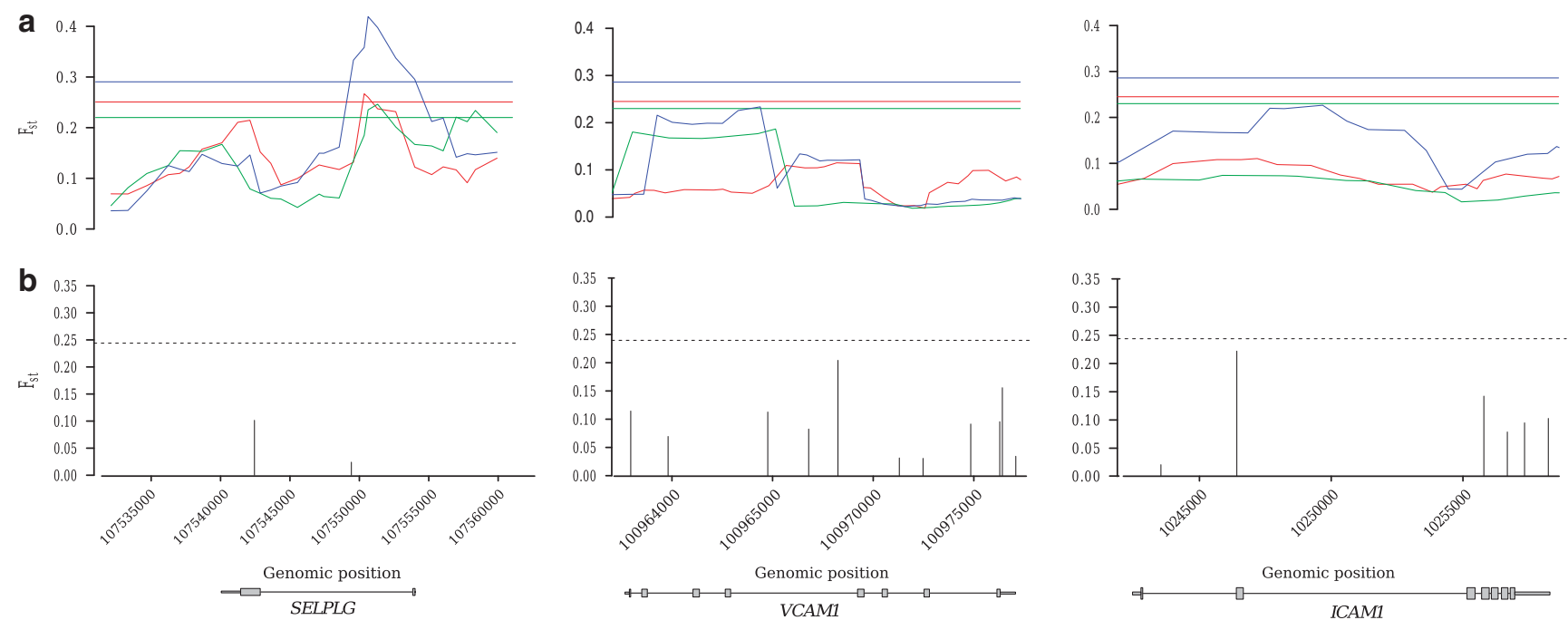

Figure 2 Sliding-window analysis of $F_{\mathrm{ST}}$ for SELPLG, ICAM1 and VCAM1. $F_{\mathrm{ST}}$ calculation and color codes as in Figure 1. (a) Data from the 1000 Genomes Pilot Project. (b) Data from the HGDP-CEPH panel.

Table 1 Nucleotide diversity and MLHKA test for the two analyzed SELP and SELPLG regions

\begin{tabular}{|c|c|c|c|c|c|c|c|c|c|c|}
\hline \multirow[t]{2}{*}{ Gene region } & \multirow[t]{2}{*}{ Size $(K b)$} & \multirow[t]{2}{*}{ Pop. } & \multirow[t]{2}{*}{$\mathrm{N}^{\mathrm{a}}$} & \multirow[t]{2}{*}{$\mathrm{S}^{\mathrm{b}}$} & \multicolumn{2}{|c|}{$\theta_{W}\left(\times 10^{-4}\right)$} & \multicolumn{2}{|c|}{$\pi\left(\times 10^{-4}\right)$} & \multicolumn{2}{|r|}{ MLHKA } \\
\hline & & & & & Value & $\operatorname{Rank}^{c}$ & Value & $\operatorname{Rankc^{c}}$ & $k^{d}$ & P-value \\
\hline \multirow[t]{3}{*}{$S E L P_{\text {ex9-10 }}$} & 6.6 & $A A$ & 48 & 37 & 12.55 & 0.81 & 11.13 & 0.81 & 1.92 & 0.11 \\
\hline & & CEU & 46 & 18 & 6.51 & 0.64 & 11.72 & 0.88 & 1.24 & 0.10 \\
\hline & & AS & 40 & 15 & 4.97 & 0.42 & 1.55 & 0.07 & 1.04 & 0.13 \\
\hline \multirow[t]{3}{*}{$S E L P_{e x 11-13}$} & 4.1 & $A A$ & 48 & 52 & 28.88 & $>0.99$ & 27.52 & 0.99 & 5.95 & $2.3 \times 10^{-6}$ \\
\hline & & CEU & 46 & 27 & 15.14 & 0.98 & 11.75 & 0.88 & 4.21 & $4.2 \times 10^{-4}$ \\
\hline & & AS & 40 & 15 & 8.69 & 0.87 & 6.12 & 0.63 & 2.83 & 0.012 \\
\hline \multirow[t]{3}{*}{$S E L P L G_{5^{\prime}}$} & 2.7 & $\mathrm{AA}$ & 48 & 18 & 15.06 & 0.91 & 10.54 & 0.79 & 2.46 & 0.062 \\
\hline & & CEU & 46 & 13 & 10.99 & 0.90 & 9.64 & 0.83 & 2.57 & 0.064 \\
\hline & & AS & 40 & 7 & 6.11 & 0.74 & 7.51 & 0.75 & 1.59 & 0.84 \\
\hline
\end{tabular}

Abbreviations: AA, African American; AS, Asian; CEU, Europeans; MLHKA, maximum-likelihood-ratio Hudson-Kreitman-Aguadé; Pop., population.

aSample size (chromosomes).
b Number of segregating sites.

'Percentile rank relative to a distribution of $5 \mathrm{~kb}$ windows from NIEHS genes.

dSelection parameter.

of polymorphism compared with divergence in all populations (Table 1). No deviation from neutrality was observed for the $S E L P_{\text {ex9-10 }}$ and SELPLG ${ }_{5}$, regions (Table 1).

As mentioned above, natural selection acting on specific gene regions can determine a distortion in the site frequency spectrum. Common neutrality tests based on the SFS include Tajima's $D\left(D_{\mathrm{T}}\right)$ (Tajima, 1989), and Fu and Li's $D^{\star}$ and $F^{\star}$ (Fu and $\mathrm{Li}, 1993$ ). $D_{\mathrm{T}}$ tests the departure from neutrality by comparing $\theta_{\mathrm{W}}$ and $\pi$, and positive values indicate an excess of intermediate frequency variants. Fu and Li's $F^{\star}$ and $D^{\star}$ are also based on the site frequency spectrum (SFS) and differ from $D_{\mathrm{T}}$, in that they also take into account whether mutations occur in external or internal branches of a genealogy. As population history, in addition to selective processes, is known to affect the SFS, we evaluated the significance of neutrality tests by performing coalescent simulations that incorporate demographic scenarios (see methods) (Schaffner et al., 2005). As explained above, we also applied an empirical comparison by calculating the percentile rank of $D_{\mathrm{T}}, F^{\star}$ and $D^{*}$ for the SELP and SELPLG gene regions relative to reference windows. No deviation from neutrality was observed in AA, whereas $D_{\mathrm{T}}$ and $F^{*}$ were significantly high in $S E L P_{\text {ex } 9-10}$ for CEU (Table 2). In this population, $D^{*}$ was extremely high in $S E L P_{\text {ex11-13. }}$. As for AS, SFS-based statistics tended to display low values in both SELP regions (Table 2). These data were confirmed by running coalescent simulations with different demographic models (Supplementary Table 1). No departure from neutrality was observed for SELPLG $G_{5^{\prime}}$.

\section{SELP haplotype analysis and selective pattern in distinct populations}

The power of SFS-based statistics to reject neutrality depends on several factors such as the timing and strength of the selective event, 
Table 2 SFS-based statistics for the analyzed SELP and SELPLG gene regions

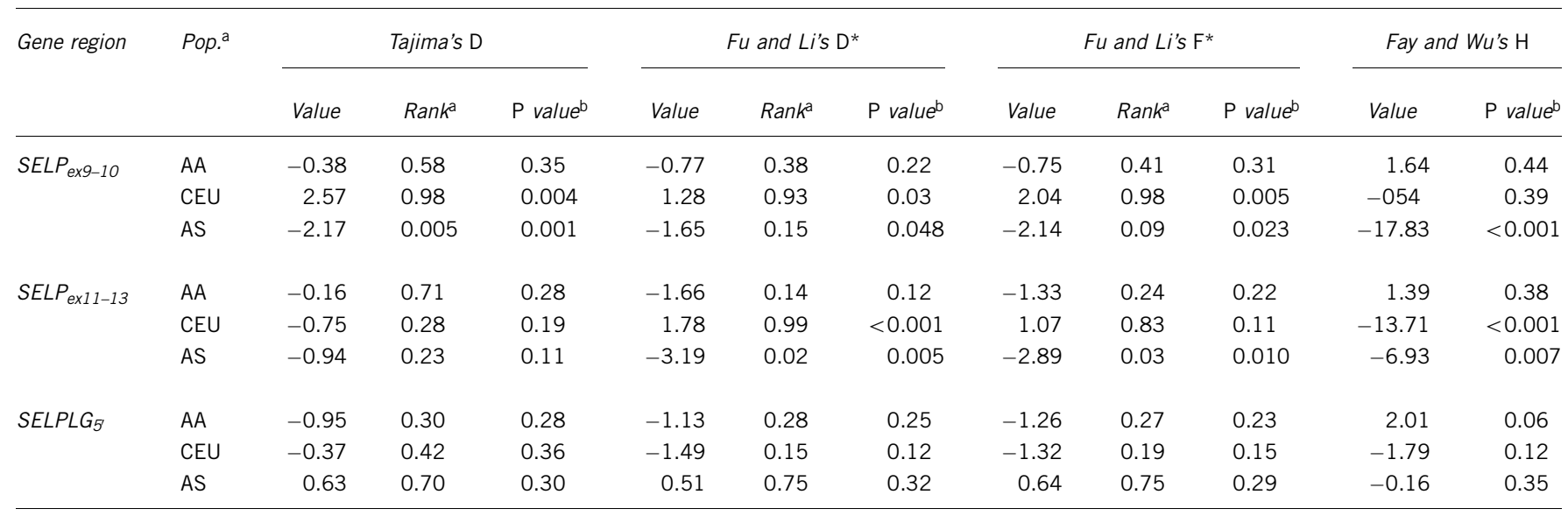

Abbreviations: AA, African American; AS, Asian; CEU, Europeans; Pop., population.

apercentile rank relative to a distribution of $5 \mathrm{~kb}$ windows from NIEHS genes.

bP-value obtained through coalescent simulations.

and complex selective patterns may leave signatures that are difficult to detect. Thus, to gain further insight into the evolutionary history of $S E L P_{\text {ex11-13, }}$, we performed haplotype analysis using two approaches: a neighbor-joining network (Bandelt et al., 1999) and a maximumlikelihood coalescent method implemented in GENETREE (Griffiths and Tavare, 1995). This latter indicated that the haplotype genealogy of SELP $P_{\text {ex11-13 }}$ is split into two major clades (clades A and B) with a coalescence time of 3.51 million years (MY) (SD: 0.569 MY) (Figure 3 ). Both clades are further split into relatively deep subclades, one of them, in clade A, showing a TMRCA of more than 2.2 MY. As shown in the haplotype network (Figure 3), no AS chromosome contributes to clade $\mathrm{B}$, whereas all other major clade A haplotypes are represented across all populations. These analyses, and the deep coalescence time of SELP $P_{\text {ex11-13 }}$ haplotypes suggest that this region has been a target of multiallelic balancing selection in CEU and AA. Conversely, the low diversity levels observed in AS for the two SELP regions indicate that a selective sweep may have occurred in this population. Indeed, calculation of Fay and Wu's $\mathrm{H}$ revealed significantly negative values for both $\operatorname{SELP}_{\text {ex9-10 }}(H=-17.83, P<0.01$, Table 2) and $\operatorname{SELP}_{\text {ex11-13 }}(H=-6.93, P=0.01$, Table 2$)$. In order to further explore this possibility, we applied the DIND test (Barreiro et al., 2009). This is based on the ratio of intra-allelic diversity associated with the ancestral and derived alleles $\left(i \pi_{\mathrm{A}} / i \pi_{\mathrm{D}}\right)$ plotted against the frequency of the derived allele (Figure 4): a high value of $i \pi_{\mathrm{A}} / i \pi_{\mathrm{D}}$ for variants with high frequency of the derived allele is suggestive of positive selection, as the neutral diversity associated with the derived allele is limited despite its high frequency in the population. We applied the DIND test to $S E L P_{\text {ex9-10 }}$ and $S E L P_{\text {ex11-13; }}$ again, statistical significance was calculated by coalescent simulations that incorporate a demographic model (see methods). Significantly, high values of DIND were obtained in SELP $P_{\text {ex9-10 }}$ for rs3917750, rs3917751, rs3766126, rs2223303, rs2205894 and rs3917777 (Figure 4). These SNPs define a haplotype with a frequency of $0.95,0.45$ and 0.10 in AS, CEU and AA, respectively. Visualization of haplotypes in this region indicated that the derived alleles of the 5 SNPs reported above define a set of highly homogeneous haplotypes in both CEU and AS, as also assessed by calculation of nucleotide diversity for the distinct haplotype groups (Figure 4).

The soluble form of P-selectin is thought to result both from alternative splicing of the transmembrane domain and from shedding of the membrane-bound form (Vestweber and Blanks, 1999). Given that the transmembrane domain is encoded by exon 14, we wished to verify whether the two major haplogroups we observed for SEL$P_{\text {ex11-13 }}$ carry variants with an effect on exon 14 inclusion in SELP transcripts. To this aim, we constructed SELP minigenes. In particular, DNA fragments spanning exons 11-16 were cloned in an expression vector. Two distinct fragments were cloned, deriving from chromosomes carrying clade A or clade B haplotypes. RT-PCR amplification of SELP transcripts isolated from HeLa cells transfected with both minigenes generated similar amounts of two products: a $433 \mathrm{bp}$ transcript deriving from the full-length SELP and a $323 \mathrm{bp}$ PCR fragment corresponding to exon 14 skipping. Albeit not quantitative, these results suggest that exon 14 skipping is not associated with a specific haplotype.

\section{Population genetics of SELP in chimpanzee}

To analyze SELP evolutionary history in Pan troglodytes, we resequenced $S E L P_{\text {ex9-10 }}$ and $S E L P_{\text {ex11-13 }}$ in nine unrelated chimpanzees. No polymorphic variant shared with humans was observed. The total number of segregating sites was 39 and 33 in $S E L P_{\text {ex9-10 }}$ and $S E L P_{\text {ex11-13 }}$, respectively; their analysis indicated that most nonsynonymous substitutions (five out of six) occur in exons 12 and 13 (Supplementary Table 2). In order to assess whether the two SELP regions show unusual levels of nucleotide variability, we resequenced 16 genomic regions in these same individuals to be used as an empirical comparison (Cagliani et al., 2012). Calculation of $\theta_{\mathrm{W}}$ that SELP $P_{\text {ex9-10 }}$ and $S E L P_{\text {ex11-13 }}$ show by far the highest values of diversity compared with the 16 reference regions (Figure 5); as for $\pi$, the value for SELP $P_{\text {ex11-13 }}$ was also the highest. This result was confirmed by application of the MLHKA test, which yielded significant results $\left(k=3.81\right.$ and $7.72, P=0.0021$ and $5.41 \times 10^{-6}$ for $S E L P_{\text {ex9-10 }}$ and $S E L P_{\text {ex11-13 }}$, respectively). Calculation of SFS statistics indicated that both SELP regions display the lowest negative values for $D_{\mathrm{T}}, D^{\star}$ and $F^{\star}$ (Supplementary Table 3). Further analysis indicated that variants in $S E L P_{\text {ex } 9-10}$ and $S E L P_{\text {ex11-13 }}$ are in tight linkage disequilibrium in the chimpanzee sample (Supplementary Figure 7), and one single individual carries a haplotype, which is highly divergent from those observed among the remaining eight primates. This translates in a haplotype genealogy with extremely long basal branches and a deep coalescent time. GENETREE analysis yielded a maximum-likelihood 


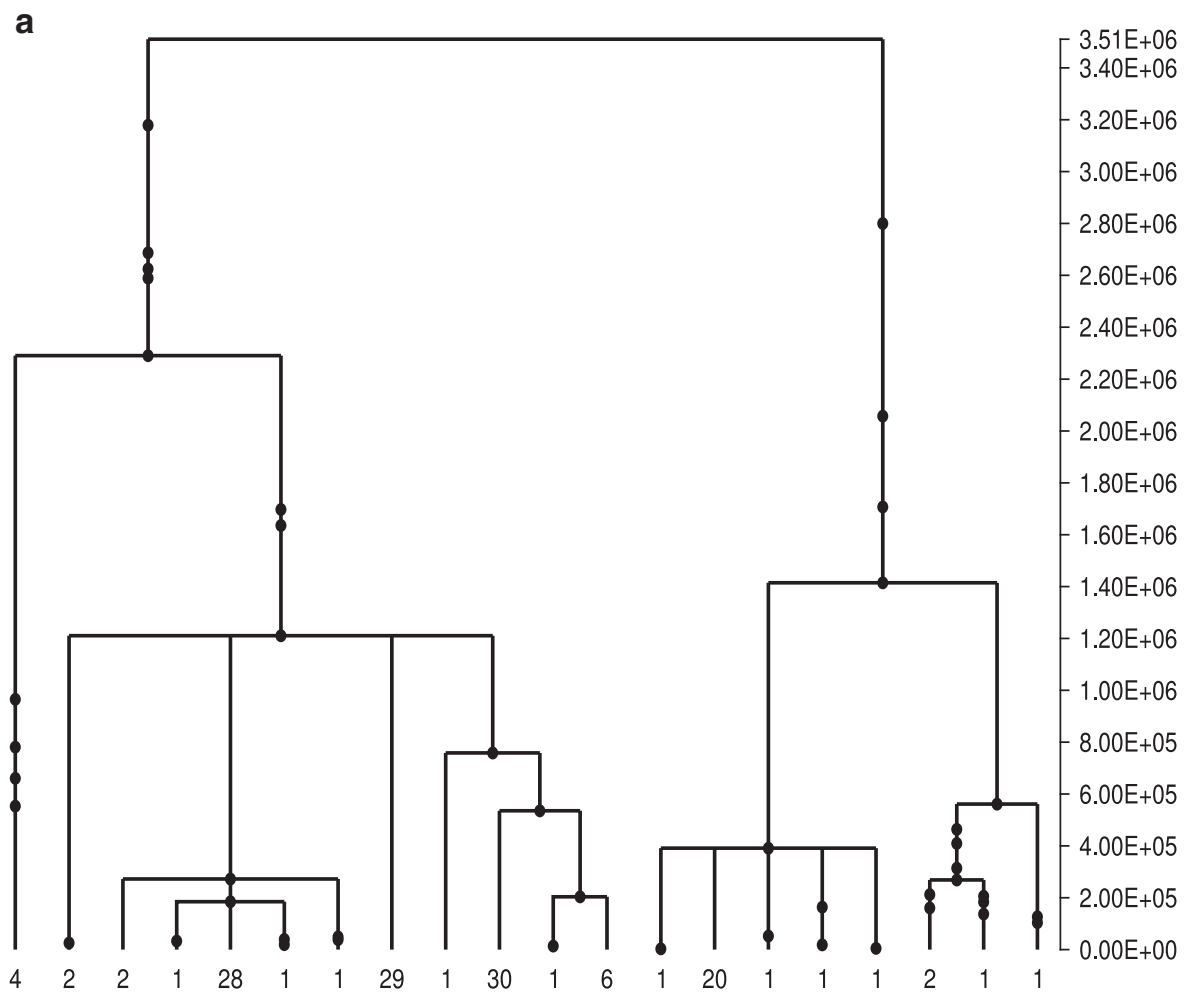

Clade A

Clade B

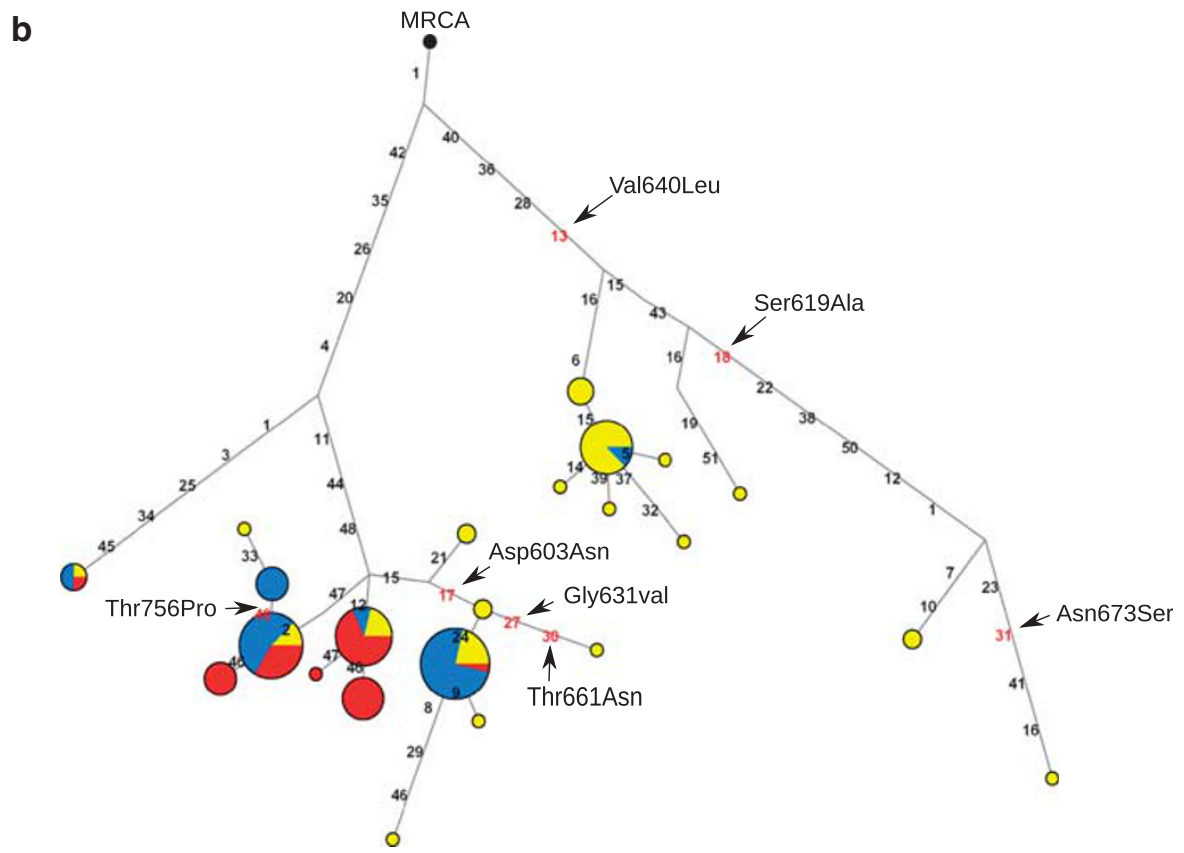

Figure 3 Haplotype analysis for SELP ex11-13. (a) GENETREE: mutations are represented as black dots. The absolute frequency of each haplotype is reported. (b) Median joining network: each node represents a different haplotype, with the size of the circle proportional to frequency. Branch lengths are proportional to the number of nucleotide differences. Circles are color-coded according to population (yellow: AA, blue: CEU, red: AS). The most recent common ancestor (MRCA) is also shown (black circle). Nonsynonymous substitutions are shown in red and indicated by the arrows.

estimate of $\theta\left(\theta_{\mathrm{ML}}\right)$ of 9.2, resulting in an effective population size $\left(\mathrm{N}_{\mathrm{e}}\right)$ of 14081. Using this method, the TMRCA of the chimpanzee SELP haplotype lineages amounted to $3.52 \mathrm{MY}$ (SD: $424 \mathrm{KY}$ ).

\section{DISCUSSION}

In this study, we analyzed the evolutionary history of genes encoding major molecules involved in leukocyte-epithelium interaction. A 
a

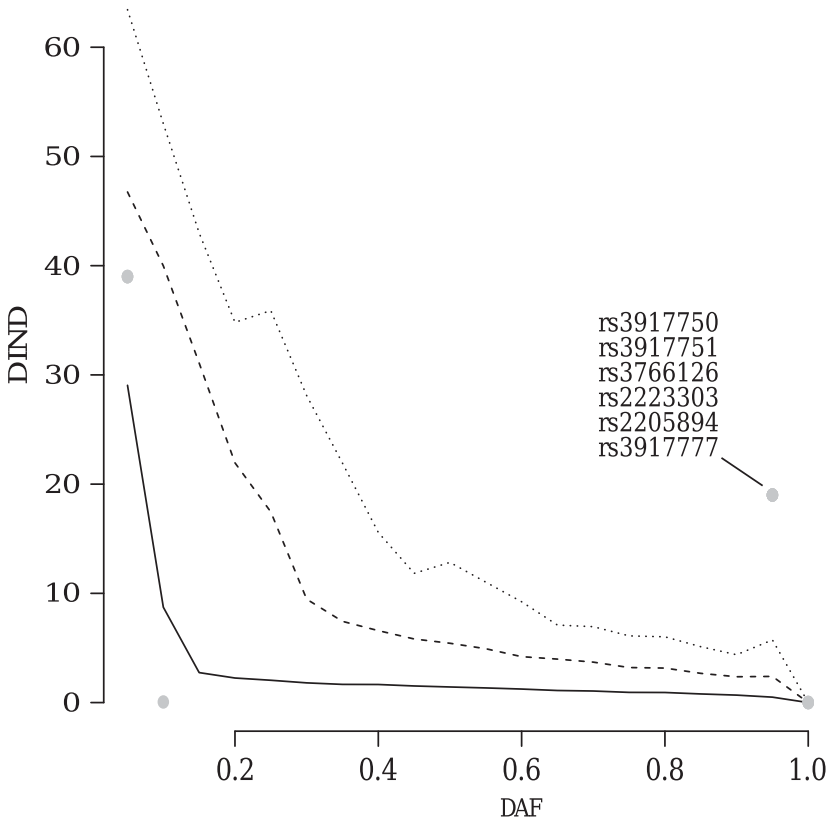

b

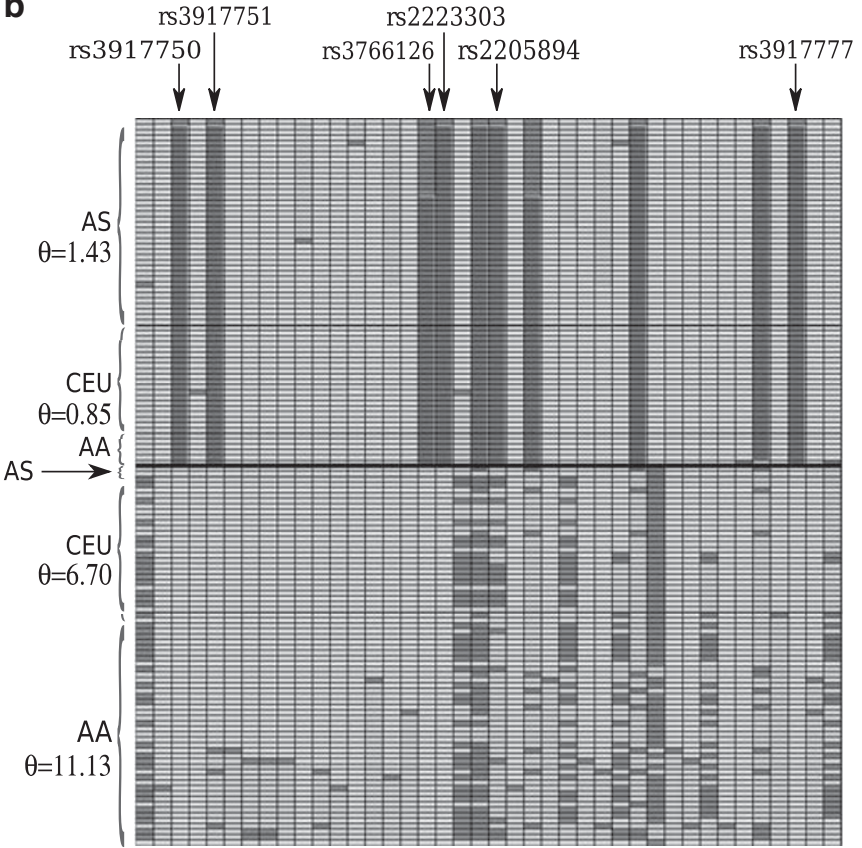

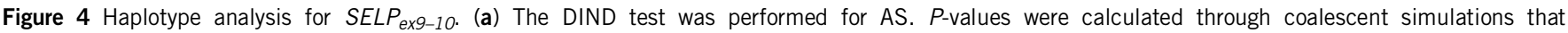
incorporate demographic scenarios (Schaffner et al., 2005). The continuous, dashed and dotted lines indicate the median, the 95th and the 99th percentiles, respectively. (b) Visualization of $S E L P_{\text {ex9-10 }}$ haplotypes; polymorphic positions are color-coded according to their allelic state (dark gray: derived, light gray: ancestral). The position of the 5 SNPs identified through the DIND test are shown and inferred haplotypes are ordered on the basis of their allelic status.

a

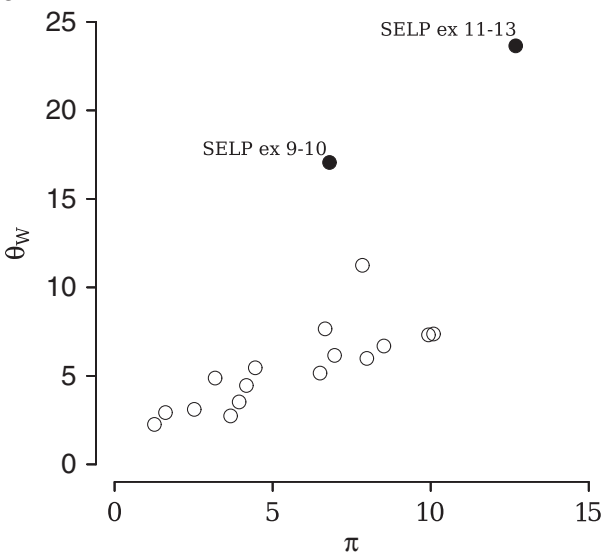

b

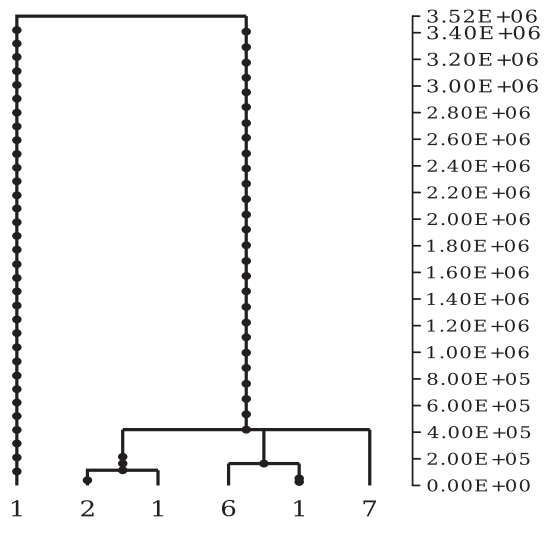

Figure 5 Nucleotide diversity and haplotype analysis in chimpanzee. (a) Plot of $\theta_{W}$ and $\pi$ values for the 16 regions we resequenced (white circles) and for the two SELP regions (black). (b) GENETREE analysis of the SELP region covering exons 9-13: mutations are represented as black dots and the absolute frequency of each haplotype is reported.

previous analysis of immune response genes with available sequences in public databases (Casals et al., 2011) included ITGAL and ITGA2, encoding leukocyte integrin molecules also involved in white blood cell trafficking; the two genes were found to be neutrally evolving. To our knowledge, no other study has addressed the evolutionary history of adhesion molecules involved in leukocyte trafficking in human populations. Here, we exploited the availability of the 1000 Genomes Project low-coverage data to perform a sliding-window analysis of $F_{\mathrm{ST}}$ along a $\sim 150 \mathrm{~kb}$ region encompassing SELL, SELE and SELP. Peaks of significantly high population genetic differentiation were identified through an empirical approach based on the comparison with a large number of human genes, and were restricted to two regions in SELP where several amino-acid changes are located. A region in SELPLG also showed high population genetic differentiation. Extremely high values of $F_{\mathrm{ST}}$ strongly suggest the action of local adaptation, as allele frequencies are driven to differ more than that would be expected on the basis of drift or demography alone. Thus, we focused on these regions, and, in order to overcome the possible biases in the SFS, which are introduced by next-generation sequencing approaches (1000 Genomes Project Consortium et al., 2010), we analyzed Sanger 
sequencing data to infer selection patterns. Results indicated that the genomic region covering $S E L P_{\text {ex11-13 }}$ displays high nucleotide diversity in Africans and CEU, and a higher level of within-species diversity compared with inter-specific divergence. Analysis of inferred haplotypes in this region revealed the presence of two deeply separated clades with a TMRCA of $\sim 3.5 \mathrm{MY}$. Coalescence times for neutrally evolving autosomal human loci range between 0.8 and 1.5 MY (Tishkoff and Verrelli, 2003); thus, the TMRCA of the SELP haplotype clades is much deeper than expected under neutrality. Indeed, clade A is further split into two lineages that coalesce at $\sim 2.2 \mathrm{MY}$ (Figure 3). Overall, these data are consistent with a model of multiallelic balancing selection and may explain the values of SFS-based statistics (Tajima's $D$ and Fu and Li's $F^{\star}$ and $D^{\star}$ ), which are not strikingly positive in $S E L P_{\text {ex11-13, }}$, as the skew toward intermediate frequency variants tends to be less marked in a multiallelic selection model than in the case of biallelic selection (Navarro and Barton, 2002). In this respect, it is worth mentioning that, although $S E L P_{\text {ex9-10 }}$ showed high values for $D_{\mathrm{T}}$ and $F^{\star}$, these features are not accompanied by a local increase in nucleotide diversity (which is a hallmark of balancing selection (Charlesworth. 2006)), suggesting that the balancing selection target(s) is located in $S E L P_{\text {ex11-13, }}$, and that linkage disequilibrium might cause the distortion of the SFS in exons 9-10. As for SELPLG, despite the high population genetic differentiation identified in the initial scan, no test suggested departure from neutrality, confirming the previous indication that high $F_{\mathrm{ST}}$ alone is not sufficient to demonstrate natural selection (Xue et al., 2009).

Network analysis of SELP $P_{\text {ex11-13 }}$ indicated that distinct haplotypes have different frequencies in the three human populations we analyzed, with AS chromosomes being absent from clade B; this explains the limited nucleotide diversity observed for this population, and suggests a different selective scenario for SELP in Asia (see below). Analysis of SNPs located on the branches of the haplotype network indicated that the Val640Leu (rs6133) polymorphism separates the two major haplotype clades and might therefore represent a selection target. The derived 640Leu allele, which is common in populations of African descent, has previously been associated with atopy, thrombo-embolic stroke and systemic lupus erythematosus, suggesting that it might affect some properties of SELP, which are relevant to immunologic/inflammatory functions (Bourgain et al., 2003; Zee et al., 2004; Morris et al., 2009). The variant has also been shown to modulate circulating levels of soluble P-selectin in some studies but not in others (Reiner et al., 2008; Barbalic et al., 2010). As for the two subclades within A group haplotypes, all variants separating the two branches are non-coding, suggesting that the selection target might be represented by one or more regulatory variants. Two nonsynonymous variants, namely Thr756Pro (rs6136) and Asp603Asn (rs6127), differentiate major haplotypes within clade A (Figure 3). The position of these variants in the haplotype genealogy indicates that they do not represent selection targets or, at least, that the selective pressure acting on them is extremely weak or recent. Nonetheless, these variants have been the subjects of intense investigation, as they were reported to affect cardiovascular functions in humans, although results have often been inconsistent among studies and in populations with different ethnic origin (Tregouet et al., 2002; Miller et al., 2004; Volcik et al., 2007). As already suggested by some authors (Tregouet et al., 2002), results herein clearly show that haplotype-based association analysis, rather than single SNP genotyping, should be applied when SELP genetic variation is to be associated with human phenotypic traits. Indeed, the SELP haplotype structure is complex and coding variants (in addition to putative regulatory variants) define distinct major haplotypes. These have extremely different frequencies in distinct human populations, also resulting in diverse statistical power to detect associations depending on sample ethnicity.

Given that a fraction of soluble P-selectin is thought to result from alternative splicing of exon 14 (Vestweber and Blanks, 1999), we also tested whether the two major haplotypes encompassing exons 11-13 might affect exon inclusion. SELP transcript levels in lymphoblastoid cell lines were low (not shown), not allowing quantification of the alternatively spliced product. For this reason we adopted a minigene approach with transfection in HeLa cells. On the one hand, this allows splicing to occur for the two haplotypes in the same conditions and genetic background, therefore removing possible confounding effects. On the other hand, a major drawback of this approach is that the minigene is expressed from an exogenous promoter, and increasing evidences suggest that polymerase elongation rate and promoter elements have an impact on alternative splicing regulation (Kornblihtt et al., 2004). Under the experimental conditions we used, the two SELP haplotypes yielded similar amounts of full-length and alternatively spliced transcripts, suggesting no major effect of $S E L$ $P_{\text {ex11-13 }}$ variants in exon 14 inclusion. However, further analyses will be required to determine whether promoter elements, environmental cues or biochemical stimuli affect the haplotype-specific splicing of SELP.

As mentioned above, striking differences in allele/haplotype frequencies, which are reflected in high $F_{\mathrm{ST}}$ values, are commonly regarded as a signature of local adaptation. Our analysis strongly suggest that while genetic diversity has long been maintained in African and CEU populations owing to a balancing selection regime, a SELP haplotype has been driven to high frequency in AS populations as a result of positive selection. Indeed, AS populations show reduced diversity at the two analyzed SELP regions, especially $S E L P_{\text {ex9-10, }}$, and neutrality tests highlight an excess of low-frequency alleles. Calculation of Fay and $\mathrm{Wu}$ (2000), which is sensitive to the presence of highfrequency-derived alleles, yielded a strongly negative result in the region encompassing exons 9-10. Consistently, application of the DIND test confirmed the hypothesis of positive selection for SELP and identified a haplotype, which has been driven to a frequency of 0.95 in AS whereas not reaching 0.5 in either CEU or AA. In both AS and CEU populations, a set of highly homogeneous haplotypes is defined by the derived alleles of the 5 SNPs we identified through the DIND test, whereas haplotypes carrying ancestral alleles at these loci display much higher diversity (Figure 4). It is worth mentioning that the two SELP regions we analyzed are located nearby, and therefore cannot be thought of evolving independently. In principle, the loss of the balanced polymorphism at $S E L P_{\text {ex11-13 }}$ in AS might explain the reduced diversity observed at $S E L P_{e x 9-10}$ in this population; yet, this scenario is not expected to result in the presence of a set of highly homogeneous haplotypes. Thus, we suggest that the selection targets in the two SELP regions have been competing, and that the selective sweep in the region encompassing exons 9-10 has determined the loss of the balanced SNP(s) in AS and reduced its frequency in CEU. In this respect, it is also worth mentioning that no significant $\mathrm{iES}$ score was detected in the region encompassing $S E L P_{\text {ex } 9-10}$; yet, simulation analyses have indicated that DIND has more power than haplotype-based tests for frequencies of the selected haplotype ranging from 10 to $98 \%$ (Barreiro et al., 2009). Variants defining the SELP $P_{\text {ex } 9-10}$ high frequency haplotype are all non-coding and inspection of available resequencing databases (for example, 1000 Genome Project; NIEHS Exome Project) indicated that they are not in linkage disequilibrium with any coding variant. Thus, the selection target in AS populations might be represented by a regulatory variant that possibly affects SELP expression. 
Overall, these data suggest that distinct human SELP variants have been targeted by natural selection possibly as a response to different environmental pressures. In an attempt to analyze the selective history of P-selectin in our closest living relatives, we sequenced the region spanning exons 9-13 in nine unrelated Pan troglodytes. Our results indicated that SELP displays extremely high diversity when compared with other loci resequenced in the same individuals. Yet, this feature is due to the presence of a single highly divergent haplotype that originates a gene phylogeny with extremely deep basal branches that coalesce at 3.5 MY. This estimated TMRCA largely pre-dates the split of the chimpanzee and bonobo lineages, as well as the dispersal of modern chimpanzee populations (Becquet et al., 2007). Thus, these data lend themselves to at least two possible explanations: either distinct SELP haplotypes have been maintained in these primates as a result of long-standing balancing selection or the ancestral primate population was structured. Indeed, archaic population structure with migration can determine the occasional sampling of loci with deep TMRCAs from extant populations. Unfortunately, the still limited knowledge of chimpanzee demographic history, and the small number of sequenced individuals complicate the interpretation of this result. Nonetheless, it is interesting to notice that in both humans and chimpanzees several amino-acid replacements tend to cluster in the region covering exons 11-13, although in either species the MK test was not significant when orangutan or macaque were used as outgroups (not shown). This gene region encodes the SELP sushi domains (also known as complement control protein modules, or short consensus repeats), which are thought to be relevant for the interaction between P-selectin and leukocytes (Ley, 2003). As mentioned above, the binding of SELP to SELPLG has a central role in mediating leukocyte tethering, rolling and extravasation to sites of acute and chronic inflammation. Thus, the selective pressure(s) acting on SELP may be related to its central role in the elicitation of immune and inflammatory responses, as infectious diseases are commonly considered a powerful selective force. SELP has been involved in the pathogenesis of severe malaria, a disease that is commonly regarded as the strongest selective pressure acting on the human genome (Pozzoli et al., 2010). P-selectin contributes to the cytoadherence of infected erythrocytes to the brain microvasculature (Rowe et al., 2009) and mice that specifically lack endothelial expression of Selp are protected against cerebral malaria (Combes et al., 2004). The parasite ligand for P-selectin is thought to be represented by PfEMP1 (Plasmodium falciparum erythrocyte membrane protein 1), as the two proteins bind in vitro (Senczuk et al., 2001). PfEMP1 is a highly variable protein which has a central role in parasite immune evasion by changing binding tropism. Thus, an interesting possibility is that the diversity of human (and possibly chimpanzee) SELP results, at least partially, from a host-pathogen genetic conflict with Plasmodium species that also accounts for local changes in allele frequency of human polymorphisms.

Immune and inflammatory responses are vital to organism survival, but need fine tuning to prevent autoimmunity and tissue damage. Interestingly, the interaction of SELP with SELPLG has been shown to contribute to the generation of tolerogenic dendritic cells (Urzainqui et al., 2007), and P-selectin binding to pentraxin 3 activates a negative feed-back loop that decreases leukocyte recruitment at inflammation sites (Deban et al., 2010). In line with these observations and with its role in immune responses, polymorphisms in SELP have been associated with autoimmune manifestations or chronic inflammatory diseases. Risk alleles for autoimmune conditions have been shown to be common targets of natural selection in humans (Sironi and Clerici, 2010), possibly because of their conferring a selective advantage against ancestral infections. This hypothesis might apply to the Val640Leu SNP in SELP, which is a likely selection target and has been associated with atopy and systemic lupus erythematosus (Bourgain et al., 2003; Morris et al., 2009). Nonetheless, data herein suggest that further genetic association studies for autoimmune diseases or other phenotypic traits would benefit from taking the complex SELP haplotype structure into account and from the analysis of possible regulatory variants in the gene, as they might have represented additional targets of natural selection.

\section{Data archiving}

Data have been deposited at Dryad: 10.5061/dryad.211vn8m4.

\section{CONFLICT OF INTEREST}

The authors declare no conflict of interest.

\section{ACKNOWLEDGEMENTS}

This work was supported by the Italian Ministry of Health (grant: RF-IEM2007-633627). We wish to thank Dr Christian Roos from the Gene Bank of Primates, Primate Genetics (Germany), for sending chimpanzee genomic DNA.

1000 Genomes Project Consortium Durbin RM, Abecasis GR, Altshuler DL, Auton A, Brooks LD, Durbin RM et al. (2010). A map of human genome variation from population-scale sequencing. Nature 467: 1061-1073.

Bandelt HJ, Forster P, Rohl A (1999). Median-joining networks for inferring intraspecific phylogenies. Mol Biol Evol 16: 37-48.

Barbalic M, Dupuis J, Dehghan A, Bis JC, Hoogeveen RC, Schnabel RB et al. (2010). Large-scale genomic studies reveal central role of ABO in SP-selectin and sICAM-1 levels. Hum Mol Genet 19: 1863-1872.

Barreiro LB, Ben-Ali M, Quach H, Laval G, Patin E, Pickrell JK et al. (2009). Evolutionary dynamics of human Toll-like receptors and their different contributions to host defense. PLoS Genet 5: e1000562.

Becquet C, Patterson N, Stone AC, Przeworski M, Reich D (2007). Genetic structure of chimpanzee populations. PLoS Genet 3: e66.

Bourgain C, Hoffjan S, Nicolae R, Newman D, Steiner L, Walker K et al. (2003). Novel case-control test in a founder population identifies $\mathrm{P}$-selectin as an atopy-susceptibility locus. Am J Hum Genet 73: 612-626.

Cagliani R, Guerini FR, Fumagalli M, Riva S, Agliardi C, Galimberti D et al. (2012). A trans-specific polymorphism in ZC3HAV1 is maintained by long-standing balancing selection and may confer susceptibility to multiple sclerosis. $\mathrm{Mol} \mathrm{Biol} \mathrm{Evol}$ 29: 1599-1613.

Casals F, Sikora M, Laayouni H, Montanucci L, Muntasell A, Lazarus R et al. (2011). Genetic adaptation of the antibacterial human innate immunity network. BMC Evol Biol 11: 202.

Charlesworth D (2006). Balancing selection and its effects on sequences in nearby genome regions. PLoS Genet 2: e64.

Combes V, Rosenkranz AR, Redard M, Pizzolato G, Lepidi H, Vestweber D et al (2004). Pathogenic role of P-selectin in experimental cerebral malaria: importance of the endothelial compartment. Am J Pathol 164: 781-786.

Deban L, Russo RC, Sironi M, Moalli F, Scanziani M, Zambelli V et al. (2010). Regulation of leukocyte recruitment by the long pentraxin PTX3. Nat Immunol 11: 328-334.

Fay JC, Wu Cl (2000). Hitchhiking under positive Darwinian selection. Genetics 155: 1405-1413.

Fu YX, Li WH (1993). Statistical tests of neutrality of mutations. Genetics 133: 693-709. Fumagalli M, Cagliani R, Pozzoli U, Riva S, Comi GP, Menozzi G et al. (2009). Widespread balancing selection and pathogen-driven selection at blood group antigen genes. Genome Res 19: 199-212.

Golias C, Tsoutsi E, Matziridis A, Makridis P, Batistatou A, Charalabopoulos K (2007). Review. Leukocyte and endothelial cell adhesion molecules in inflammation focusing on inflammatory heart disease. In Vivo 21: 757-769.

Griffiths RC, Tavare S (1995). Unrooted genealogical tree probabilities in the infinitelymany-sites model. Math Biosci 127: 77-98.

Jacob CO, Reiff A, Armstrong DL, Myones BL, Silverman E, Klein-Gitelman M et al. (2007). Identification of novel susceptibility genes in childhood-onset systemic lupus erythematosus using a uniquely designed candidate gene pathway platform. Arthritis Rheum 56: 4164-4173.

Kornblihtt AR, de la Mata M, Fededa JP, Munoz MJ, Nogues G (2004). Multiple links between transcription and splicing. RNA 10: 1489-1498.

Ley $\mathrm{K}$ (2003). The role of selectins in inflammation and disease. Trends Mol Med 9: 263-268. 
Miller MA, Kerry SM, Dong Y, Strazzullo P, Cappuccio FP (2004). Association between the Thr715Pro P-selectin gene polymorphism and soluble P-selectin levels in a multiethnic population in South London. Thromb Haemost 92: 1060-1065.

Morris DL, Graham RR, Erwig LP, Gaffney PM, Moser KL, Behrens TW et al. (2009). Variation in the upstream region of P-Selectin (SELP) is a risk factor for SLE. Genes Immun 10: 404-413.

Navarro A, Barton NH (2002). The effects of multilocus balancing selection on neutral variability. Genetics 161: 849-863.

Nei M, Li WH (1979). Mathematical model for studying genetic variation in terms of restriction endonucleases. Proc Natl Acad Sci USA 76: 5269-5273.

Pozzoli U, Fumagalli M, Cagliani R, Comi GP, Bresolin N, Clerici M et al. (2010). The role of protozoa-driven selection in shaping human genetic variability. Trends Genet 26 : 95-99.

Reiner AP, Carlson CS, Thyagarajan B, Rieder MJ, Polak JF, Siscovick DS et al. (2008). Soluble P-selectin, SELP polymorphisms, and atherosclerotic risk in EuropeanAmerican and African-African young adults: the Coronary Artery Risk Development in Young Adults (CARDIA) Study. Arterioscler Thromb Vasc Biol 28: 1549-1555.

Rowe JA, Claessens A, Corrigan RA, Arman M (2009). Adhesion of Plasmodium falciparum-infected erythrocytes to human cells: molecular mechanisms and therapeutic implications. Expert Rev Mol Med 11: e16.

Schaffner SF, Foo C, Gabriel S, Reich D, Daly MJ, Altshuler D (2005). Calibrating a coalescent simulation of human genome sequence variation. Genome Res 15 1576-1583.

Senczuk AM, Reeder JC, Kosmala MM, Ho M (2001). Plasmodium falciparum erythrocyte membrane protein 1 functions as a ligand for P-selectin. Blood 98: 3132-3135.

Sironi M, Clerici M (2010). The hygiene hypothesis: an evolutionary perspective. Microbes Infect 12: 421-427.

Stephens M, Scheet $P$ (2005). Accounting for decay of linkage disequilibrium in haplotype inference and missing-data imputation. Am J Hum Genet 76: 449-462.

Tajima $F$ (1989). Statistical method for testing the neutral mutation hypothesis by DNA polymorphism. Genetics 123: 585-595.
Tang K, Thornton KR, Stoneking M (2007). A new approach for using genome scans to detect recent positive selection in the human genome. PLOS Biol 5: e171.

Tishkoff SA, Verrelli BC (2003). Patterns of human genetic diversity: implications for human evolutionary history and disease. Annu Rev Genomics. Hum Genet 4: 293-340.

Tregouet DA, Barbaux S, Escolano S, Tahri N, Golmard JL, Tiret L et al. (2002). Specific haplotypes of the P-selectin gene are associated with myocardial infarction. Hum Mol Genet 11: 2015-2023.

Urzainqui A, Martinez del Hoyo G, Lamana A, de la Fuente H, Barreiro O, Olazabal IM et al. (2007). Functional role of $P$-selectin glycoprotein ligand 1/P-selectin interaction in the generation of tolerogenic dendritic cells. J Immunol 179: 7457-7465.

Vestweber D, Blanks JE (1999). Mechanisms that regulate the function of the selectins and their ligands. Physiol Rev 79: 181-213.

Volcik KA, Ballantyne CM, Coresh J, Folsom AR, Boerwinkle E (2007). Specific P-selectin and $\mathrm{P}$-selectin glycoprotein ligand-1 genotypes/haplotypes are associated with risk of incident CHD and ischemic stroke: the Atherosclerosis Risk in Communities (ARIC) study. Atherosclerosis 195: e76-e82.

Watanabe Y, Inoue T, Okada H, Kotaki S, Kanno Y, Kikuta T et al. (2006). Impact of selectin gene polymorphisms on rapid progression to end-stage renal disease in patients with IgA nephropathy. Intern Med 45: 947-951.

Watterson GA (1975). On the number of segregating sites in genetical models without recombination. Theor Popul Biol 7: 256-276.

Wright S (1950). Genetical structure of populations. Nature 166: 247-249.

Wright SI, Charlesworth B (2004). The HKA test revisited: a maximum-likelihood-ratio test of the standard neutral model. Genetics 168: 1071-1076.

Xue Y, Zhang X, Huang N, Daly A, Gillson CJ, Macarthur DG et al. (2009). Population differentiation as an indicator of recent positive selection in humans: an empirical evaluation. Genetics 183: 1065-1077.

Zee RY, Cook NR, Cheng S, Reynolds R, Erlich HA, Lindpaintner K et al. (2004). Polymorphism in the P-selectin and interleukin-4 genes as determinants of stroke: a population-based, prospective genetic analysis. Hum Mol Genet 13: 389-396.

Supplementary Information accompanies the paper on Heredity website (http://www.nature.com/hdy) 